\title{
Suppressing Interference from Noise in Sound Source Localization with Distributed Multisensor Array
}

\author{
Shengjia Zheng, ${ }^{1,2}$ Wenjie Zheng, ${ }^{1}$ Jian Shu, ${ }^{1}$ Tiejun Li, ${ }^{1}$ \\ Chin-Cheng Chen, ${ }^{1,3^{*}}$ and Fanzhu Jin ${ }^{4 * *}$ \\ ${ }^{1}$ College of Information Engineering, Jimei University, Xiamen 361021, China \\ ${ }^{2}$ Institute of Acoustics, Chinese Academy of Sciences, Beijing 100190, China \\ ${ }^{3}$ Department of Aeronautical Engineering, Chaoyang University of Technology, Taichung 413310, Taiwan \\ ${ }^{4}$ College of Mathematics and Information Science, Longyan University, Fujian 364000, China
}

(Received December 31, 2019; accepted June 19, 2020)

Keywords: matched field processing, distributed multisensor array, localization, multiple-constraint noise suppression

To suppress interference from discrete noise of underwater sound sources, we apply matched field processing with multiple-constraint noise suppression (MNS MFP) to a distributed multisensor array. Optimal weight vectors and coherent processing are also combined with MNS MFP. The results from simulations clearly show the effectiveness of the method to localize and differentiate noise and sound sources. The probability of successful sound source localization (SSL) for the new method is higher than 0.9 with an error range of $5 \%$ for depth and distance. In addition, it accurately locates a sound source and a noise source at the same distance from the multisensory array at a depth difference of $33 \mathrm{~m}$. This localization ability is much improved from that of the conventional matched field processing (CMFP) and diagonal loading minimum variance distortionless response (DL-MVDR) methods. With further development, the proposed method is expected to provide more accurate and precise data for underwater acoustics studies.

\section{Introduction}

Sonar systems often encounter problems in detecting targets with strong interference and noise. Ma et al. proposed a discrete noise suppression method by matching field processing (MFP) with a single towed line array. ${ }^{(1)}$ Han et al. suggested the use of spatial matrix filter technology to cancel the vessel noise. ${ }^{(2)}$ However, using a single array of sensors and filtering noise do not provide satisfactory results owing to background noise and interference.

Sound source localization (SSL) requires signal processing of data from underwater acoustic arrays, usually with MFP. MFP matches the amplitude and phase of a sound source with an underwater sound field model, which estimates the distance and depth of the sound source using data from multisensory arrays. ${ }^{(3-5)}$ The complexity of the marine environment reduces and sometimes completely eliminates the ability to locate a sound source in the case of 
discrete interference by noise sources near the target sound source. ${ }^{(6,7)}$ The suppression of the interference to obtain the sound from the source requires multiple sensor nodes in a distributed multisensor array system, The number of spatial samplings of a system can be increased to further improve the reliability of underwater SSL. ${ }^{(8-10)}$ We propose to apply MFP technology combined with multiple-constraint noise suppression (MNS) using a distributed multisensor array. To suppress discrete noise more effectively, we establish an optimization problem and solve it to minimize the variance of the MFP output under the condition that there is no interfered (distorted) beam response in the observed area. The optimization problem is solved using the Lagrange multiplier method. MFP with MNS enables SSL to be achieved accurately in underwater acoustic channels with interference and noise from their sources.

\section{Methods}

\subsection{MFP with distributed multisensor array}

MFP processes sound data received by hydrophones to match the data with a replica field vector predicted by a mathematical model. A hydrophone in a multisensor array acquires underwater acoustic signals. It generates an output power proportional to the acoustic pressure generated by the signals. The hydrophone has a series of piezoelectric ceramic sheets with the same polarity. A sound pressure signal triggers the piezoelectric ceramic sheets to generate an output power by bending vibration. The output power indicates the depth and distance of a sound source processed by MFP and is defined as

$$
P(\varphi)=\boldsymbol{w}^{H}(\varphi) \boldsymbol{K} \boldsymbol{w}(\varphi)
$$

where $\boldsymbol{w}$ is a weight vector, ( $)^{H}$ is the conjugate transpose, $\varphi=\left[f, z_{r}, z_{s}, r\right]$ is a location parameter of the sound source, $f$ is the sound source signal processing frequency, $z_{s}$ is the depth of the sound source, $z_{r, j}$ is the depth of the $j$ th element on the receiving array, $r$ is the horizontal distance between the sound source and the array, and $\boldsymbol{K}$ is the cross-spectral density matrix.

Conventional matched field processing (CMFP) is less sensitive than adaptive MFP (AMFP) in differentiating signals of a real sound source from those of an interference source, especially with sparse spatial sampling. CMFP has more side lobes than AMFP.

The CMFP weight vector is expressed as

$$
\boldsymbol{w}_{C M F P}(\varphi)=\frac{\boldsymbol{v}(\varphi)}{\boldsymbol{v}^{H}(\varphi) \boldsymbol{v}(\varphi)}
$$

where $\boldsymbol{v}(\varphi)=[v(\varphi), v(\varphi), \ldots, v(\varphi)]^{\mathrm{T}}$ is the replica field vector of the array and $\boldsymbol{v}^{H}(\varphi) \boldsymbol{v}(\varphi)=N(N$ : number of array elements).

AMFP is an algorithm and uses minimum variance distortionless response (MVDR) constraints. The output power of AMFP has effectively suppressed side lobes and interference. 
In practice, to ensure the reversibility of the cross-spectral density matrix and the robustness of environmental parameter mismatches, white Gaussian noise is added to a matched processor of diagonal loading minimum variance distortionless response (DL-MVDR). ${ }^{(11)}$ The weight vector of DL-MVDR is

$$
\boldsymbol{w}_{D L-M V D R}(\varphi)=\frac{\left(\boldsymbol{K}+\delta_{d}^{2} \boldsymbol{I}\right)^{-1} \boldsymbol{v}(\varphi)}{\boldsymbol{v}^{H}(\varphi)\left(\boldsymbol{K}+\delta_{d}^{2} \boldsymbol{I}\right)^{-1} \boldsymbol{v}(\varphi)},
$$

where $\boldsymbol{I}$ is the unit matrix and $\delta_{d}$ is the diagonal loading factor.

CMFP and AMFP basically process data from a single node, that is, generally a single towed array or a single vertical array. Higher gain can be acquired by the coherent processing of data from a distributed multisensor array system. Multiple nodes of the distributed multisensor array system have multipath propagation and coherent properties in underwater acoustic channels. They yield better estimation of the location of a sound source. Figure 1 shows a schematic diagram of localization by a distributed multisensor array system with three vertical arrays.

The output power of MFP with a distributed multisensor array is calculated as

$$
P=\boldsymbol{w}^{H} \boldsymbol{K}_{x} \boldsymbol{w}=\sum_{l=1}^{3} \boldsymbol{w}_{l}^{H} \boldsymbol{K}_{l l} \boldsymbol{w}_{l}+\sum \sum_{i, j=1, i \neq j}^{3} \boldsymbol{w}_{i}^{H} \boldsymbol{K}_{i j} \boldsymbol{w}_{j},
$$

where $\boldsymbol{K}_{l l}$ is the single-node cross-spectral density matrix and $\boldsymbol{K}_{i j}$ is the multimode crossspectral density matrix. The weight vectors $\boldsymbol{w}_{C M P F}$ and $\boldsymbol{w}_{D L-M V D R}$ allow MFP with CMFP and DL-MVDR to localize sound sources. However, a way to suppress interference and noise is required. Thus, this study tests a new discrete noise suppression method for a distributed multisensor array system.

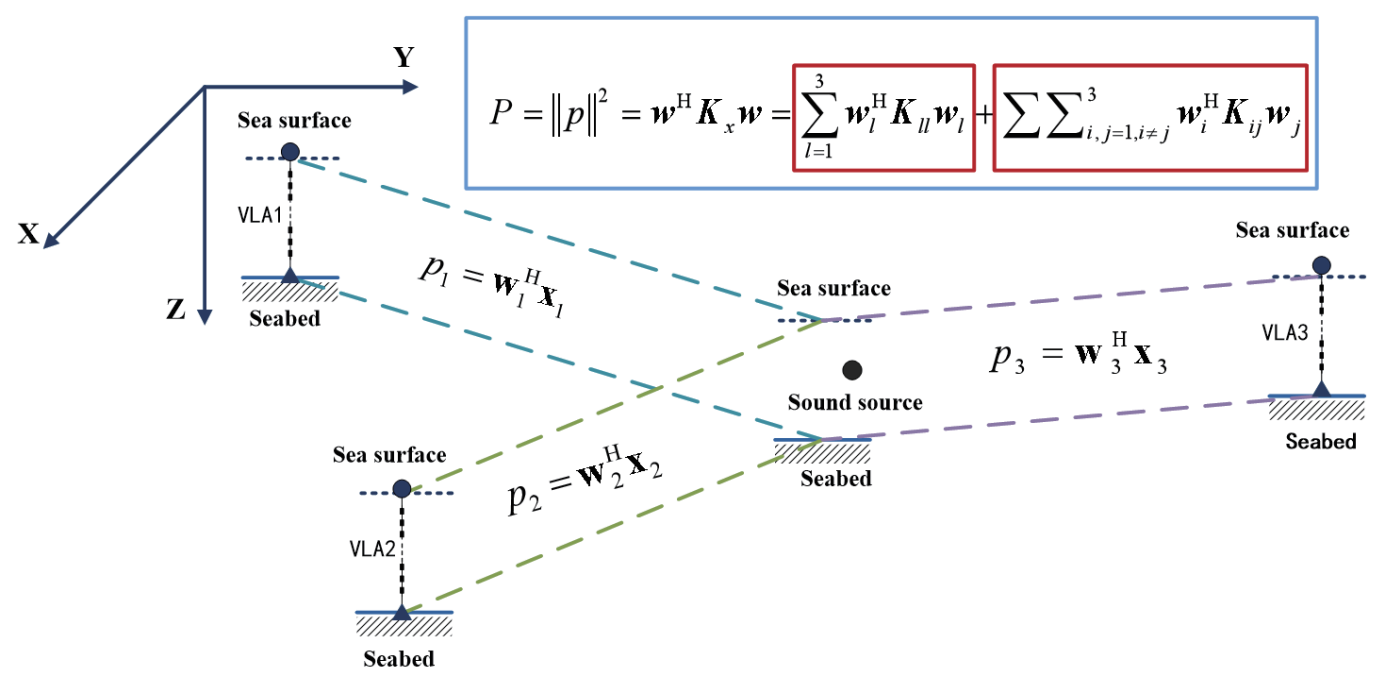

Fig. 1. (Color online) Schematic diagram of localization by distributed multisensor array system. 


\subsection{MNS MFP}

Assuming that there is one sound source and $M-1$ interference sources, the constraint on the interference is defined as

$$
\boldsymbol{w}^{H} \boldsymbol{\alpha}_{1}(\varphi)=d_{1}, \ldots, \boldsymbol{w}^{H} \boldsymbol{\alpha}_{M}(\varphi)=d_{M},
$$

where $\boldsymbol{\alpha}_{1}(\varphi)$ is the weight vector of the observed sound source, $\boldsymbol{\alpha}_{2}(\varphi), \boldsymbol{\alpha}_{3}(\varphi)$, and $\boldsymbol{\alpha}_{M}(\varphi)$ are replica field vectors obtained by the model calculation of $M-1$ interference sources, and $\left[d_{1}, d_{2}, \ldots, d_{M}\right]$ is the constraint vector. From this, an optimization problem and the minimum mean square error of the output power of MFP are calculated as

$$
\begin{gathered}
\min _{\boldsymbol{w}}[P(\varphi)]=\min \left[\boldsymbol{w}^{H}(\varphi) \boldsymbol{K} \boldsymbol{w}(\varphi)\right] \\
\text { s.t. }\left\{\begin{array}{c}
\boldsymbol{w}^{H} \boldsymbol{\alpha}_{1}(\varphi)=d_{1} \\
\vdots \\
\boldsymbol{w}^{H} \boldsymbol{\alpha}_{M}(\varphi)=d_{M}
\end{array}\right.
\end{gathered}
$$

Then, we construct the Lagrange function as

$$
L\left(\boldsymbol{w}, \lambda_{1}, \lambda_{2}, \ldots, \lambda_{M}\right)=\boldsymbol{w}^{H} \boldsymbol{K} \boldsymbol{w}-\sum_{m=1}^{M}\left[\lambda_{m}\left(\boldsymbol{w}^{H} \boldsymbol{\alpha}_{m}-d_{m}\right)\right]
$$

where $\lambda_{M}$ is a Lagrange multiplier. If we define the following equations:

$$
\begin{gathered}
\boldsymbol{E}=\left[\boldsymbol{\alpha}_{1}(\varphi)\left|\boldsymbol{\alpha}_{2}(\varphi)\right| \cdots \mid \boldsymbol{\alpha}_{M}(\varphi)\right], \\
\lambda=\left[\begin{array}{llll}
\lambda_{1} & \lambda_{2} & \cdots & \lambda_{M}
\end{array}\right]^{\mathrm{T}}, \\
\boldsymbol{d}=\left[\begin{array}{llll}
d_{1} & d_{2} & \cdots & d_{M}
\end{array}\right],
\end{gathered}
$$

then Eq. (5) becomes

$$
\boldsymbol{w}^{H} \boldsymbol{E}=\boldsymbol{d} .
$$

To find the partial derivative of Eq. (7) and make the result equal to zero, the following equation is needed. 


$$
\frac{\partial L(\boldsymbol{w}, \lambda)}{\partial \boldsymbol{w}^{*}}=2 \boldsymbol{K} \boldsymbol{w}+\boldsymbol{E} \lambda=0
$$

Then, the optimal weight vector is obtained by

$$
\boldsymbol{w}_{M N S}=\boldsymbol{K}^{-1} \boldsymbol{E}\left[\boldsymbol{E}^{H} \boldsymbol{K}^{-1} \boldsymbol{E}\right]^{-1} \boldsymbol{d}^{H}
$$

As a result, MFP for noise suppression has the optimal weight vector $\boldsymbol{w}_{M N S}$ that has a constant response to the observed signal while suppressing discrete interference and noise from the sources. In conclusion, MNS MFP efficiently suppresses discrete interference and noise in underwater acoustics channels.

\subsection{Environment model and simulation method}

We performed computer simulations to verify the effectiveness of the proposed MNS MFP. Figure 2 shows a diagram of the distributed multisensor array system with three nodes used in the simulation. Each node comprises 50 hydrophones in a vertical array with a spacing of $2 \mathrm{~m}$ at a depth of $100 \mathrm{~m}$. The three nodes form an equilateral triangle whose sides have a length of $1 \mathrm{~km}$.

The horizontal distance to two sources (noise source and sound source) from the coordinate origin of the detection system is $8 \mathrm{~km}$. The depths of the noise source and sound source are 7 and $40 \mathrm{~m}$, respectively, and the signals from the sources are band-limited white Gaussian noise with a center frequency of $300 \mathrm{~Hz}$. The signal-to-noise ratio is $-10 \mathrm{~dB}$. The environment sound profile has a weak positive gradient, and the parameters of the model are shown in Fig. 3.

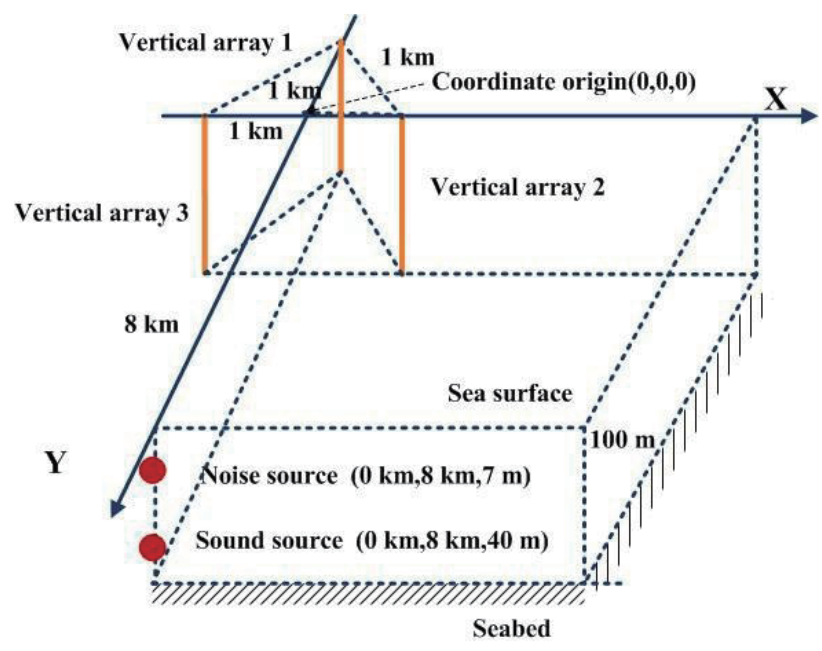

Fig. 2. (Color online) Schematic diagram of distributed multisensor array system and locations of sound and noise sources. 


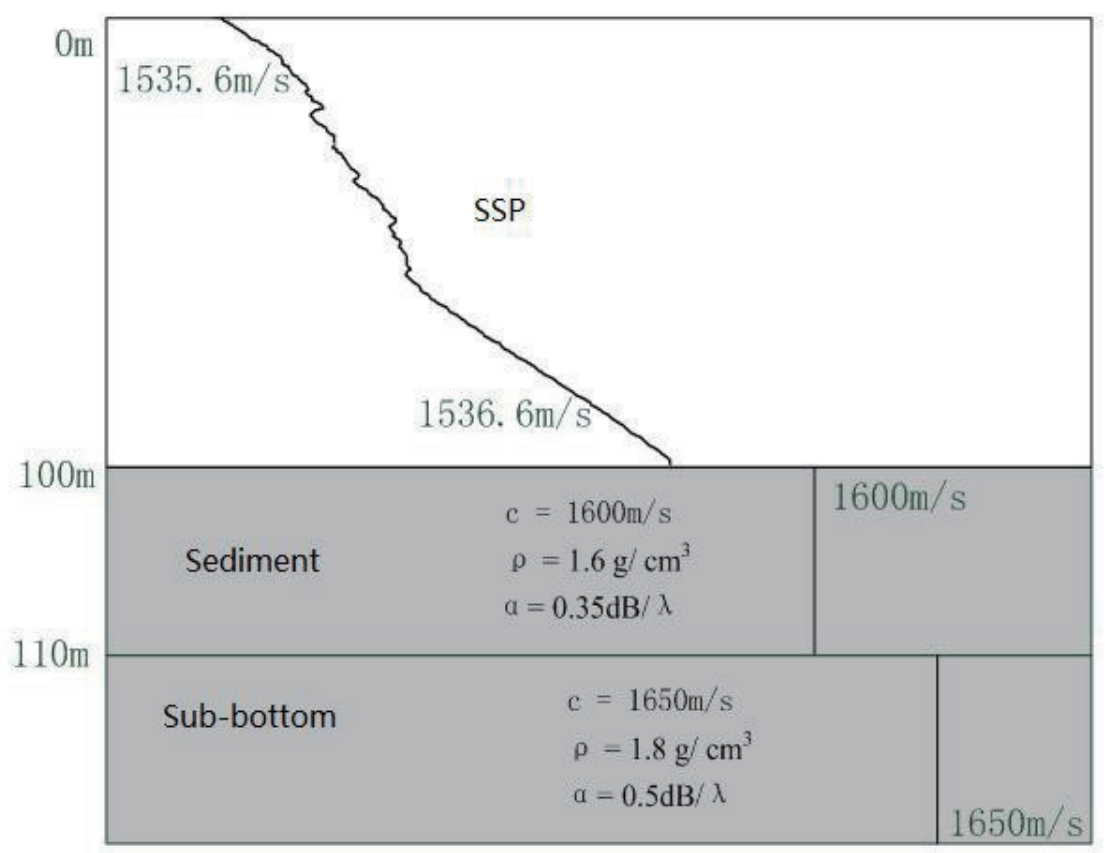

Fig. 3. Environment model for the simulation for MNS MFP.

\section{Results and Discussion}

\subsection{Localization of sound and noise sources in vertical array 1}

Figure 4 shows distributions of the sound field intensity from the two sources at depths of 7 and $40 \mathrm{~m}$ obtained by computer simulation using Matlab ${ }^{\circledR}$ software.

Figure 5 shows results of SSL of vertical array 1 with CMFP and DL-MVDR MFP projected to the coordinate origin. The result for the output power shows that the two methods had limited effectiveness in locating the underwater sound source with interference from the noise source, which is masked by side lobes. CMFP only located the noise source at the depth of $7 \mathrm{~m}$, and had a very wide range of depth to localize the source accurately. In addition, intense interference was observed. DL-MVDR had much less interference. However, it only located the noise source with high probability and could not find the sound source at all.

To apply the proposed method (MNS MFP) in this study, we designed the optimal weight vector of the response to be as small as possible to locate the noise source. Figure 6 shows the output power of MNS MFP, which located the sound source successfully and completely suppressed the interference from the noise source in vertical array 1. The result does not show any interference and clearly locates the sound source at a depth of $40 \mathrm{~m}$. In addition, MNS MFP does not reveal any signal from the noise source. 


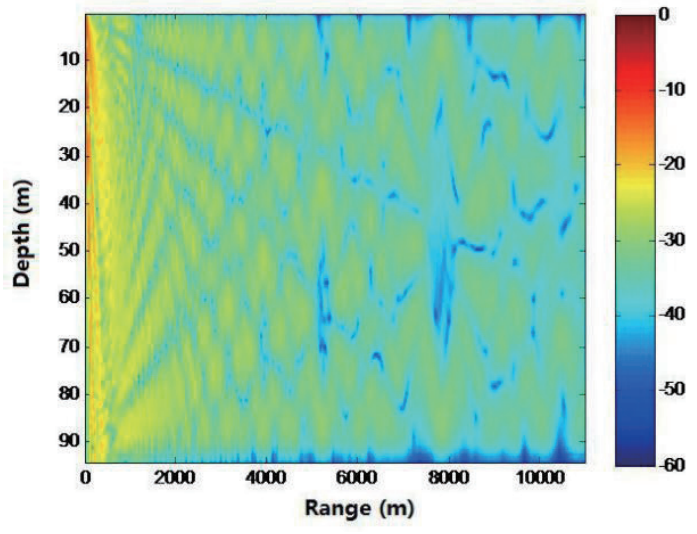

(a)

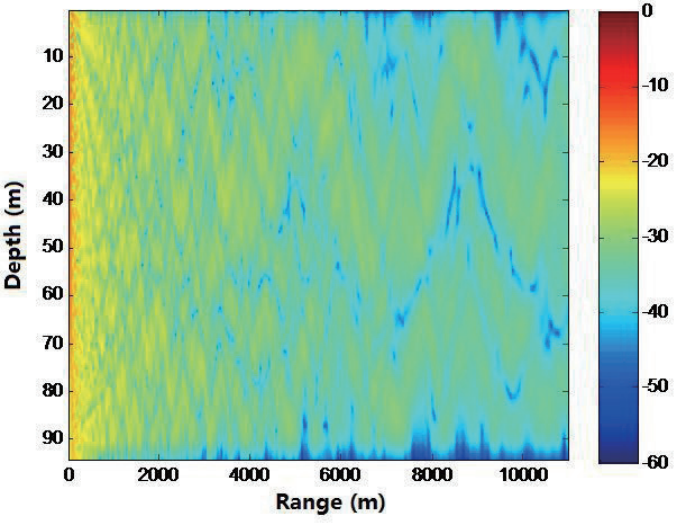

(b)

Fig. 4. (Color online) Sound field intensity distribution (the bars on the right show the intensity in dB). (a) Noise source at $7 \mathrm{~m}$ depth and (b) sound source at $40 \mathrm{~m}$ depth.

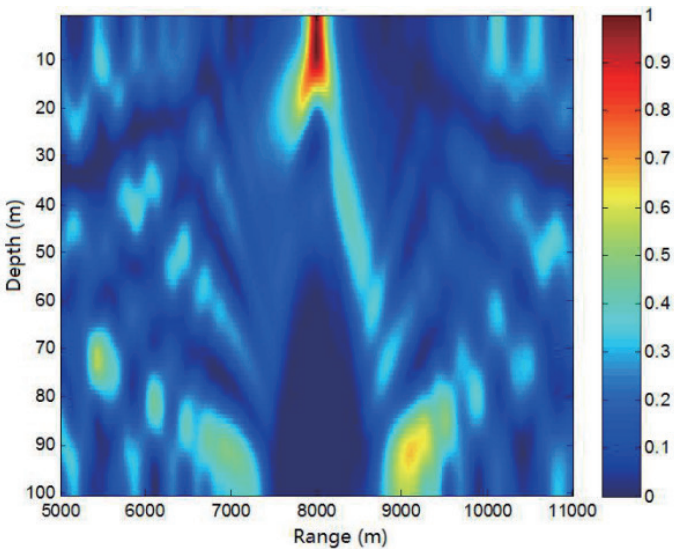

(a)

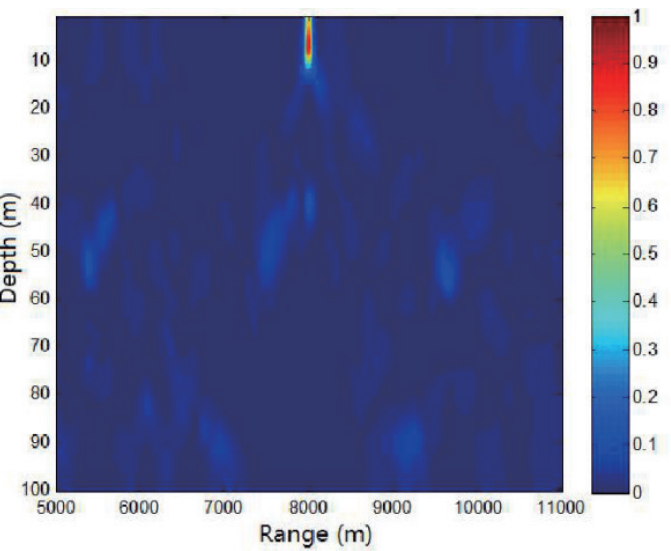

(b)

Fig. 5. (Color online) Localization results of vertical array 1 using CMFP and DL-MVDR (the bars on the right show probability). (a) CMFP. (b) DL-MVDR.

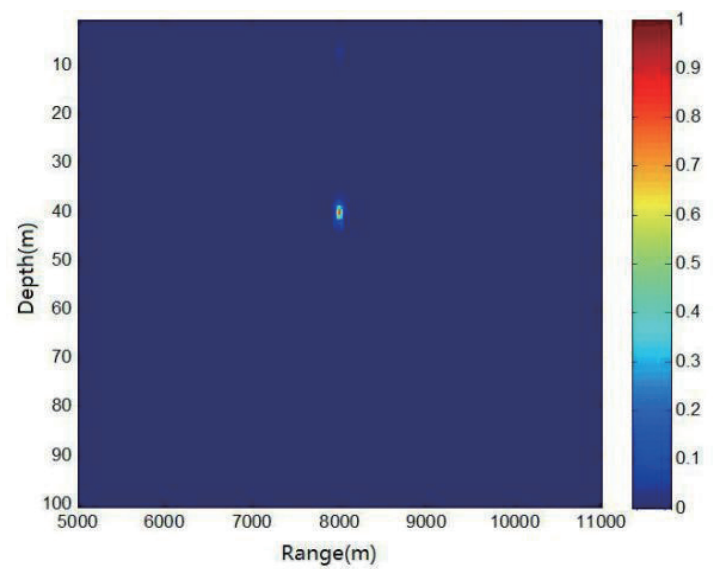

Fig. 6. (Color online) Localization results of vertical array 1 using the MFP with MNS (the bar on the right shows probability). 


\subsection{Localization of sound and noise sources in distributed multisensory array system}

Figure 7 shows the localization results of CMFP and DL-MVDR MFP for the distributed multisensor array system. The intensity of the noise was $10 \mathrm{~dB}$ stronger than that of the sound. The results show similar localization performance to that for vertical array 1. CMFP located the two sources but with intense interference. However, CMFP had a higher probability of localization for the noise source than for the sound source. DL-MVDR had much less interference, but only located the noise source, with no sign of the sound source appearing.

When we used the suppression method of MNS MFP proposed in this paper, which used a distributed multisensor array system, the underwater sound source was accurately located. Figure 8 reveals that the interference was completely suppressed for the distributed multisensor array system. In addition, MNS MFP even localized the two sources with much higher probability for the sound source.

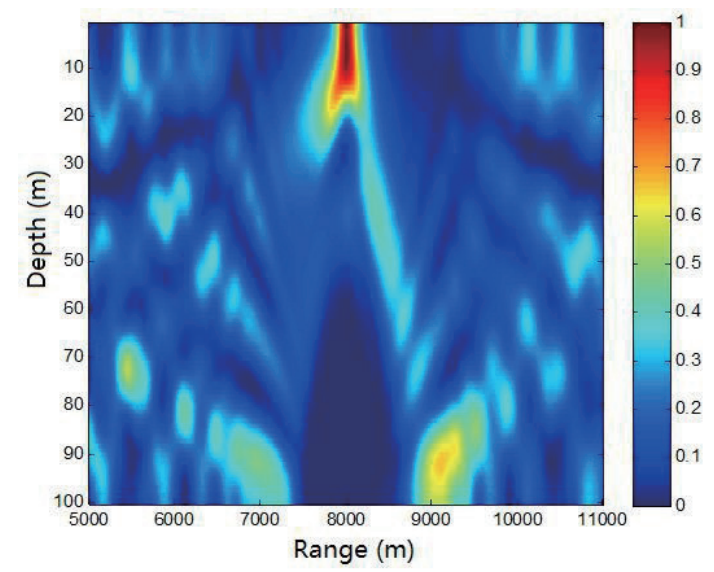

(a)

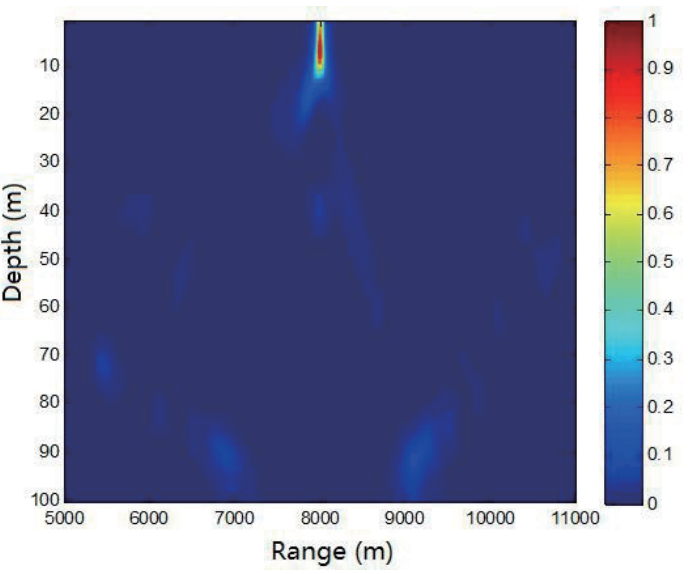

(b)

Fig. 7. (Color online) Localization results of distributed multisensor array system using CMFP and DL-MVDR (the bars on the right show probability). (a) CMFP and (b) MFP with DL-MVDR.

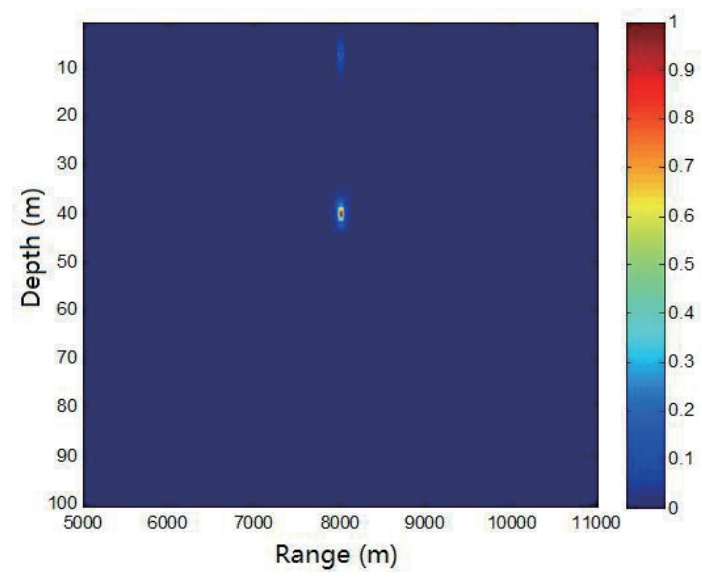

Fig. 8. (Color online) Localization results of distributed multisensor array system using MNS MFP (the bars on the right show probability). 


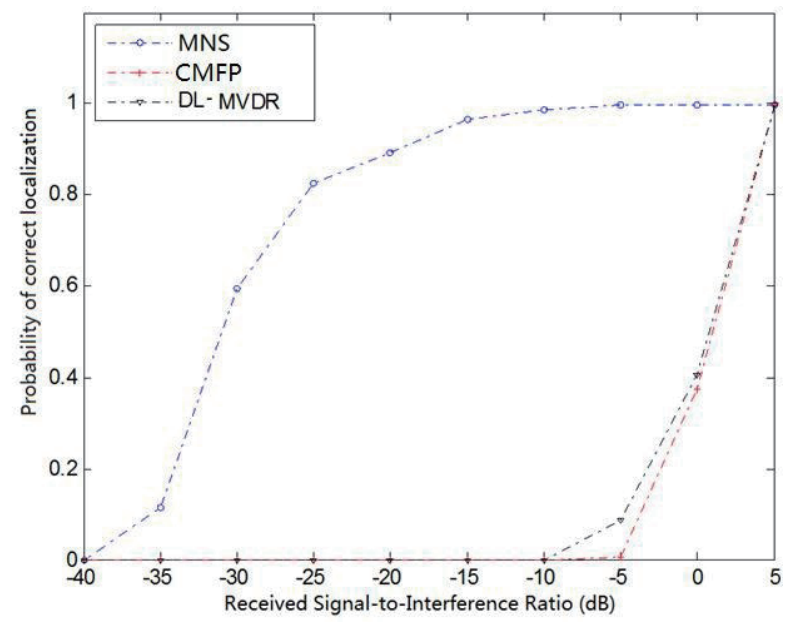

Fig. 9. Probability of correct localization under different signal-to-interferences ratios.

\subsection{Comparison of MNS MFP with CMFP and DL-MVDR}

We calculated the probability of correct localization of the sound source by three methods under different signal-to-interference ratios. When the error of estimation of the sources is within $5 \%$ (for depth and distance), the localization is considered to be successful. We performed 50 identical and independent experiments with CMFP, DL-MVDR, and MNS MFP to localize the noise and sound sources. Figure 9 shows the probability of correct localization of the sound source by the methods. When the signal-to-interference ratio was $-5 \mathrm{~dB}, \mathrm{CMFP}$ and DL-MVDR could not locate the underwater sound source. In contrast, MNS MFL could locate the sources even though it had lower probability for correct localization than CMFP and DL-MVDR. When the signal-to-interference ratio was $-5,-10$, or $-15 \mathrm{~dB}$, MNS MFP had a probability close to 1 .

\section{Conclusions}

We proposed a new method of removing interference from noise sources to localize the sound source in underwater acoustic channels by combining MFL with MNS. In addition, we optimized MNS MFL with the optimal weight vector and coherent processing for the distributed multisensor array. The simulation results verified the effectiveness of the proposed method in localizing the sound source by removing interference from the noise source. A comparison of the results of MNS MFL, CMFP, and DL-MVDR confirmed that the method localizes the sound source accurately at various signal-to-interference ratios. In particular, the method has a higher probability than 0.9 to localize the source for a signal-to-interference ratio higher than $-15 \mathrm{~dB}$. This implies that MNS MFP suppresses the interference from any noise effectively and achieves the accurate localization of an underwater sound source. 


\section{Acknowledgments}

This work was supported by the Provincial Natural Science Foundation of Fujian, China (under Grants 2016J01668 and 2017J01764). It was also supported by the Foundation of Education Department of Fujian Province under Grant JA15264 and the Foundation of Jimei University under Grant ZQ2014013.

\section{References}

1 Y. Ma, S. Yan, and K. Yang: Chin. Sci. Bull. 48 (2003) 1207. https://doi.org/10.1007/BF03183938

2 D. Han, X. H. Zhang, and Y. Sun: Acta Acustica. 36 (2011) 405. https://doi.org/10.15949/ j.cnki.0371-0025.2011.04.005

3 C. Soares, S. M. Jesus, and E. Coelho: J. Acoust. Soc. Am. 122 (2007) 3391. https://doi.org/10.1121/1.2799476

4 K. Yang, N. R. Chapman, and Y. Ma: J. Acoust. Soc. Am. 121 (2007) 833. https://doi.org/10.1121/1.2427125

5 C. F. Huang, P. Gerstoft, and W. S. Hodgkiss: J. Acoust. Soc. Am. 123 (2008) 197. https://doi.org/10.1121/1.290 8406

6 Q. H. Li, S. Q. Li, C. Y. Sun, and H. B. Yu: Acta Acustica. 32 (2007) 1. https://doi.org/10.15949/j.cnki.0371-0025.2 007.01 .001

7 E. K. Westwood: J. Acoust. Soc. Am. 91 (1992) 2777. https://doi.org/10.1121/1.402958

8 J. C. Chen, L. Yip, and J. Elson:Proc. IEEE. 91 (2003) 1154. https://doi.org/10.1109/jproc.2003.814924

9 A. Nehorai and E. Paldi: IEEE Trans. Signal Process. 42 (1994) 2481. https://doi.org/10.1109/78.317869

10 A. Hassanien, S. A. Elkader, A. B. Gershman, and K. M. Wong: IEEE Trans. Signal Process. 54 (2006) 1587. https://doi.org/10.1109/tsp.2006.870564

11 C. S. MacInnes: IEEE J. Oceanic Eng. 29 (2004) 488. https://doi.org/10.1109/joe.2004.827290

\section{About the Authors}

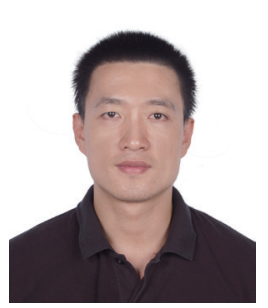

Shengjia Zheng received his B.S. degree from Jimei University, China, in 2002, his M.S. degree from Huaqiao University, China, in 2008, and his Ph.D. degree from the Institute of Acoustics, Chinese Academy of Sciences, China, in 2014. From 2014 to 2017, he was an assistant professor at Jimei University, China. Since 2018, he has been an associate professor at Jimei University. His research interests are in array signal processing, adaptive filtering, and sensors. (zhengshengjia@jmu.edu.cn)

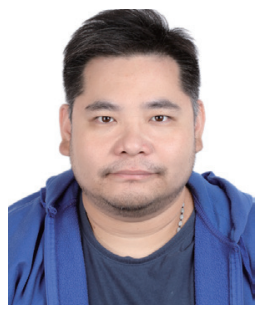

Wenjie Zheng received his B.S. degree from Jimei University, China, in 2004 and his M.S. degree from Xiamen University, China, in 2010. From 2004 to 2018, he was an assistant professor at Jimei University, China. His research interests are in embedded systems, software modeling, and sensors.

(cin911@jmu.edu.cn) 


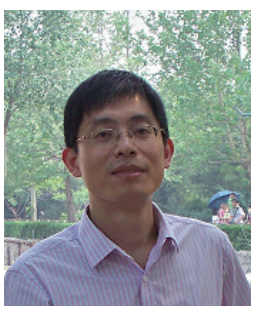

Jian Shu received his B.S. degree in electrical engineering from East China Jiaotong University, Nanchang, China, in 2000 and his M.S. degree in system engineering from Lausitz University of Applied Sciences, Senftenberg, Germany, in 2003. He has been a lecturer at the Information Engineering College, Jimei University, China, since 2004. His research interests are in sensors, measurement technologies, and underwater acoustical signal processing. (jianseecn@qq.com)

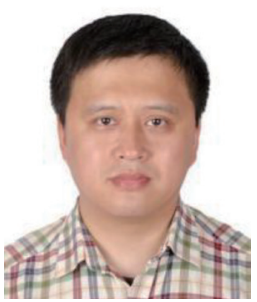

Tiejun Li received his B.S., M.S., and Ph.D. degrees from Chongqing University, China, in 2000, 2005, and 2018, respectively. From 2008 to 2014, he was an assistant professor at Jimei University, China. Since 2015, he has been an associate professor at Jimei University. His research interests are in terahertz science, artificial intelligence, and sensors. (litiejun@jmu.edu.cn)

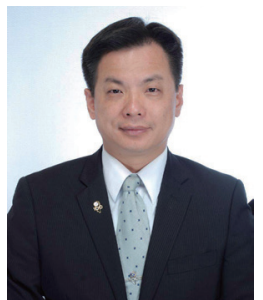

Chin-Cheng Chen has been a professor at Jimei University, China, since 2017. He became a member of IEEE in 2011 and a senior member in 2016. He earned his M.S. and Ph.D. degrees from the Department of Mechatronics Engineering, National Changhua University of Education. He has been studying RFID applications in various fields of industry. His research interests include AIoT technology and RFID applications.

(201761000018@jmu.edu.cn)

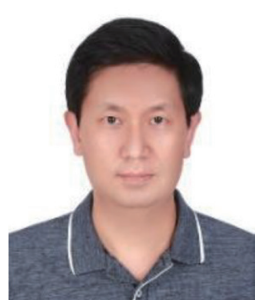

Fanzhu Jin received his Ph.D. degree from Seoul National University, Republic of Korea, in 2001. He then started working at Gdansk Medical University as an assistant professor. He is affiliated with the College of Mathematics and Information Engineering of Longyan University, Fujian, China. His research interests are in IoT and its applications. 